\title{
Effect of maternal smoking during pregnancy on Tourette syndrome and chronic tic disorders among offspring: a systematic review and meta-analysis
}

\author{
Erfan Ayubi, PhD ${ }^{1,2}$, Kamyar Mansori, PhD³, Amin Doosti- Irani, PhD ${ }^{4}$ \\ ${ }^{1}$ Health Promotion Research Center, ${ }^{2}$ Department of Community Medicine, School of Medicine, Zahedan University of Medical Sciences, Zahedan; \\ ${ }^{3}$ Department of Biostatistics and Epidemiology, School of Medicine, Zanjan University of Medical Sciences, Zanjan; ${ }^{4}$ Department of Epidemiology, \\ School of Public Health and Research Center for Health Sciences, Hamadan University of Medical Sciences, Hamadan, Iran
}

Current studies have controversial reports about the effect of maternal smoking during pregnancy (MSDP) on the risk of psychiatric disorders among offspring. The purpose of this systematic review and meta-analysis was to assess the effect of MSDP on the risk of Tourette syndrome (TS) and chronic tic disorders (CTD) among offspring. A systematic search using PubMed, Scopus, and Web of Science was conducted up to July 2019 to retrieve potential studies in English. Fixed and random-effects meta-analyses was performed to estimate pooled relative risk (RR) with $95 \%$ confidence interval $(\mathrm{Cl})$ according to heterogeneity. The methodological quality of the included studies was evaluated using the Newcastle-Ottawa Scale. Heterogeneity among studies was explored using the $\mathrm{I}^{2}$ statistic. Of the 641 studies from the initial search, 7 were included in the quantitative synthesis ( 3 cohort and 4 case control studies). Overall, MSDP increased the risk of TS and CTD among the offspring by $35 \%$ (pooled RR, 1.35; 95\% Cl, 1.17-1.56; $I^{2}=45.8 \%$; $P$-value $=0.08$ ). The results of this meta-analysis revealed that there is a significant association between MSDP and the risk of TS and CTD in offspring, and MSDP may be considered a potential risk factor for TS and CTD.

Keywords: Tic disorders; Tourette syndrome; Smoking; Prenatal

\section{Introduction}

Tourette syndrome (TS) and chronic tic disorders (CTD) are 2 neurodevelopmental disorders, which are usually diagnosed during childhood [1]. The prevalence of TS and CTD has been estimated to range from 2.6 to 38 and 3 to 50 per 1,000 children, respectively [2]. The prevalence of these disorders among boys is higher than that in girls [3]. These conditions affect the health status, familial and social relationships, education, employment, and daily life of patients [2].

Based on the results of genetic and familial studies, both TS and CTD are heritable conditions [4-6]; however, environmental factors are also important in the etiology of both TS and CTD [7]. Environmental factors such as smoking during pregnancy, pregnancy complications, low birth weight $[8,9]$, and infection [10] have been shown to be associated with these disorders.

To date, several studies have assessed the effect of maternal smoking during pregnancy (MSDP) on the risk of TS and
CTD among offspring $[8,11,12]$; however, there is controversy among the results of these studies. Therefore, this systematic review and meta-analysis aimed to estimate the association between MSDP and the risk of TS and CTD among offspring.

Received: 2020.08.22. Revised: 2020.10.20. Accepted: 2020.11.08. Corresponding author: Amin Doosti-Irani, PhD

Department of Epidemiology, School of Public Health and Research Center for Health Sciences, Hamadan University of Medical Sciences, Hamadan 6517838736, Iran

E-mail: a_doostiirani@yahoo.com

https://orcid.org/0000-0003-0623-7503

Articles published in Obstet Gynecol Sci are open-access, distributed under the terms of the Creative Commons Attribution Non-Commercial License (http://creativecommons. org/licenses/by-nc/3.0/) which permits unrestricted non-commercial use, distribution, and reproduction in any medium, provided the original work is properly cited.

Copyright $\odot 2021$ Korean Society of Obstetrics and Gynecology 


\title{
Obstetrics \& Gynecology Science
}

\author{
Vol. 64, No. 1, 2021
}

\section{Methods}

The present systematic review and meta-analysis were performed according to the Preferred Reporting Items for Systematic Reviews and Meta-Analyses (PRISMA) statement [13].

\section{Search strategy}

The literature evaluating the association between MSDP and TD/CTD disorders was systematically searched in the 3 electronic databases - PubMed, Scopus, and Web of Sciences, up to July 2019 without any limitations on type of document, time, or publication status. The following keywords were used in combination or alone: "Tourette syndrome", "tic disorders", "tics", "Tourette", "chronic tic", "smoking", "parental smoking", "maternal smoking", "pregnancy" and "child". The search strategy used for each database is presented in detail in Appendix 1.

\section{Study selection}

The initial search was imported into Endnote software (version X6; Thomson Reuters, Toronto, Canada). After removing duplications, all potential documents were independently scanned by 2 authors (EA and KM) and in cases of disagreement, the 2 authors discussed the article and came to a consensus.

\section{Eligibility criteria}

The eligible articles were retrieved by evaluating the titles and abstracts of the identified studies, though the full text was screened if necessary. All observational studies, including cohort, case control, and cross-sectional studies, that evaluated the association between MSDP and TD/CTD were included which met the following criteria: 1) reported crude or adjusted relative risk and 95\% confidence intervals (Cls) for the association between MSDP and TS/CTD, or 2) provided the original data about the association for estimating relative risk. Animal or in vitro studies, reviews, case studies, correspondences, editorials, book reviews, and conference or meeting abstracts were excluded.

\section{Data extraction}

The following items were extracted from each study: the name of the first author, year of publication, study design, country, type of study, outcome, classification system for diagnosis of the outcome, age, gender, MSDP levels, number of cases and controls (exposed to MSDP and non-exposed), source of control and matching variables for case control studies, crude and adjusted hazard ratio (HR), relative risk (RR) or odds ratio (OR) with $95 \% \mathrm{Cls}$, and adjusted confounders. We selected one specific adjusted RR from each study for the adjusted RRs to be independent and mutually exclusive in the meta-analysis.

\section{Assessment the risk of bias}

The Newcastle-Ottawa Scale (NOS) was used to assess the risk of bias. This scale consists of the following main domains: selection, comparability, exposure, and outcome [14]. A study was classified as high quality (low risk of bias) when it received 6 or more stars.

\section{Statistical analysis}

Statistical analyses were performed using Stata SE version 11 (StataCorp, College Station, TX, USA). The outcome of interest was expected to be rare among both groups, i.e., exposed to MSDP and unexposed, whereby RR was approximated by HRs and ORs [15]. Heterogeneity among studies was evaluated using the $\mathrm{I}^{2}$ estimate; heterogeneity was considered as low, moderate, and high when $\mathrm{I}^{2}$ values were $<25 \%, 25-50 \%$, and $>50 \%$, respectively. An $\mathrm{I}^{2}$ value higher than $50 \%$ was considered a substantial and large heterogeneity [16]. In case of high heterogeneity among studies, summary relative measures from each study were combined using random-effects models, which incorporate heterogeneity in the weighted average of relative measure. However, both fixed-effect and random-effects analyses were considered to reveal the presence of small-study effects. Fixed-effect and random-effects analyses were performed using the inverse variance method. The potential publication bias was assessed using funnel plots, Begg's test, and Egger's test. $P<0.05$ was considered significant.

\section{Results}

\section{Study inclusion and characteristics of included studies}

The PRISMA flow diagram of the study selection is shown in Fig. 1. Initial searches yielded 641 citations, and after removing duplications and screening the titles and abstracts, 9 articles met the eligibility criteria $[11,12,17-23]$. Two stud- 


\title{
Obstetrics \& Gynecology Science
}

\author{
Erfan Ayubi, et al. MSDP and TS, CTD and TS/CTD
}

ies $[17,20]$ were not included in the final analysis because one of them [17] reported a mean difference in the number of patients with tic disorder according to prenatal smoking exposure (yes vs. no), and another study [20] reported a linear regression coefficient for the association between prenatal maternal smoking and increased symptom severity in TS. Finally, 7 observational studies [11,12,18,21-24] provided relative risks and were considered for quantitative metaanalysis.

The characteristics of these 7 studies are presented in Table 1. Of the included studies, 3 were cohort studies $[11,18,21]$ consisting of $3,106,024$ children, including 8,258 with TS or CTD. The 4 remaining were case control designs [12,22-24], with 205 exposed to MSDP among 1,073 TS/CTD cases. The sample of included studies varied largely in size, from 153 [24] to $3,026,861$ [18]. The identified TS/CTD cases in the studies were predominantly among male patients with age under 15 years. The list of adjusted covariates in each study is given in Table 1.

\section{Risk of bias assessment}

Overall, all included studies were of good quality and had

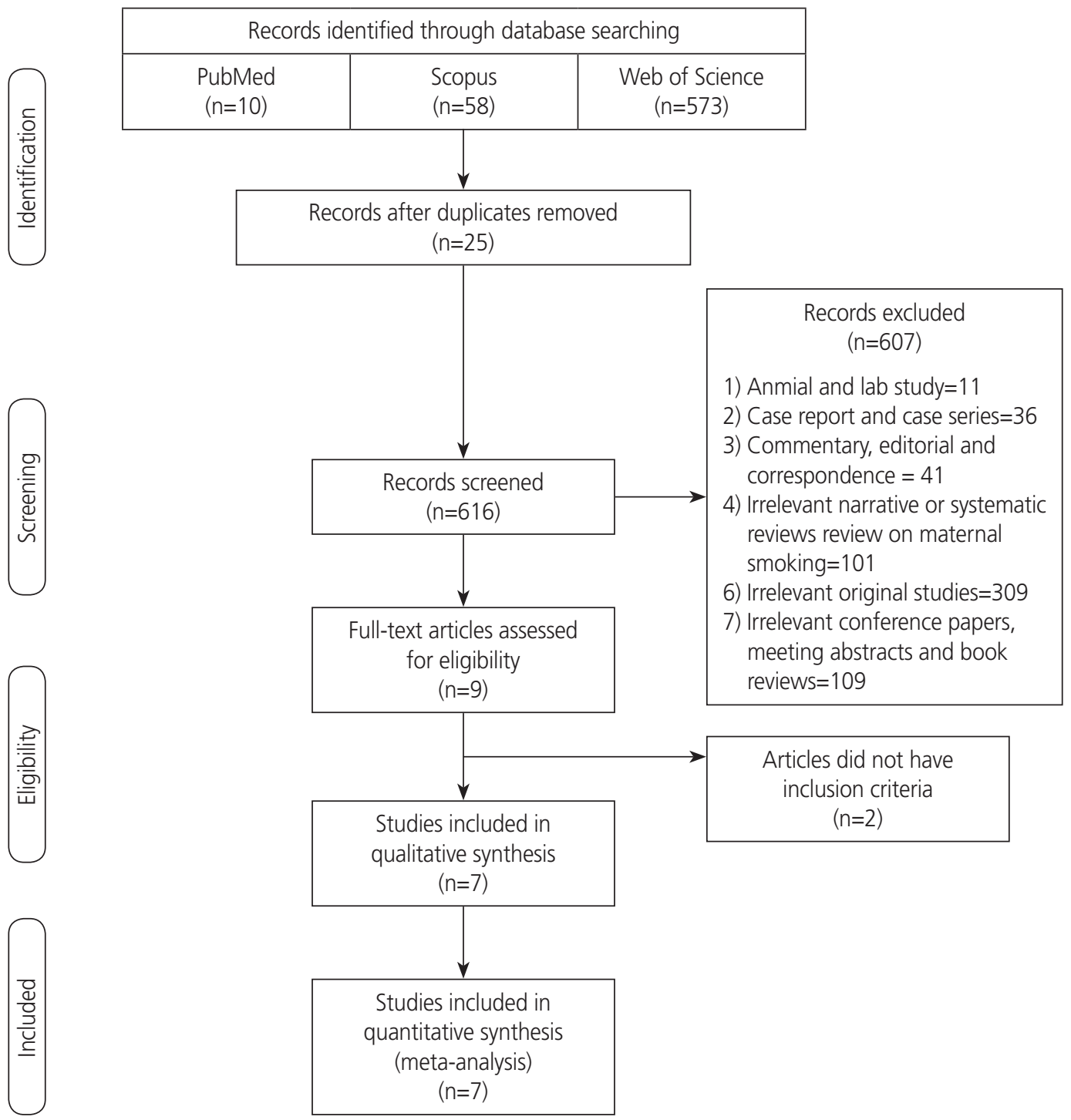

Fig. 1. Preferred Reporting Items for Systematic Reviews and Meta-Analyses (PRISMA) flow diagram of the study selection process for a systematic review and meta-analysis of the association between maternal smoking during pregnancy (MSDP) and the risk of Tourette syndrome and chronic tic disorders. 


\section{Obstetrics \& Gynecology Science}

Vol. 64, No. 1, 2021

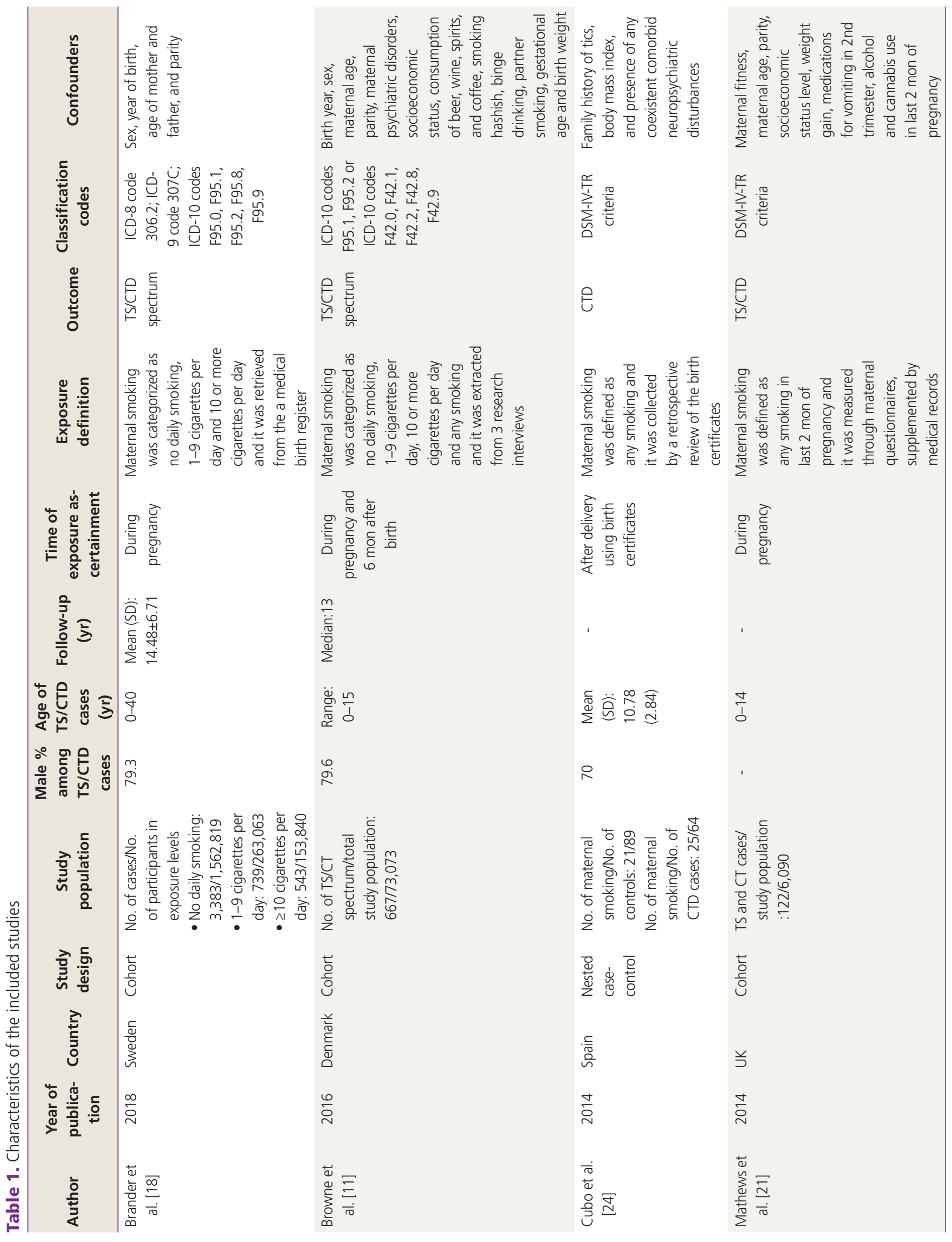




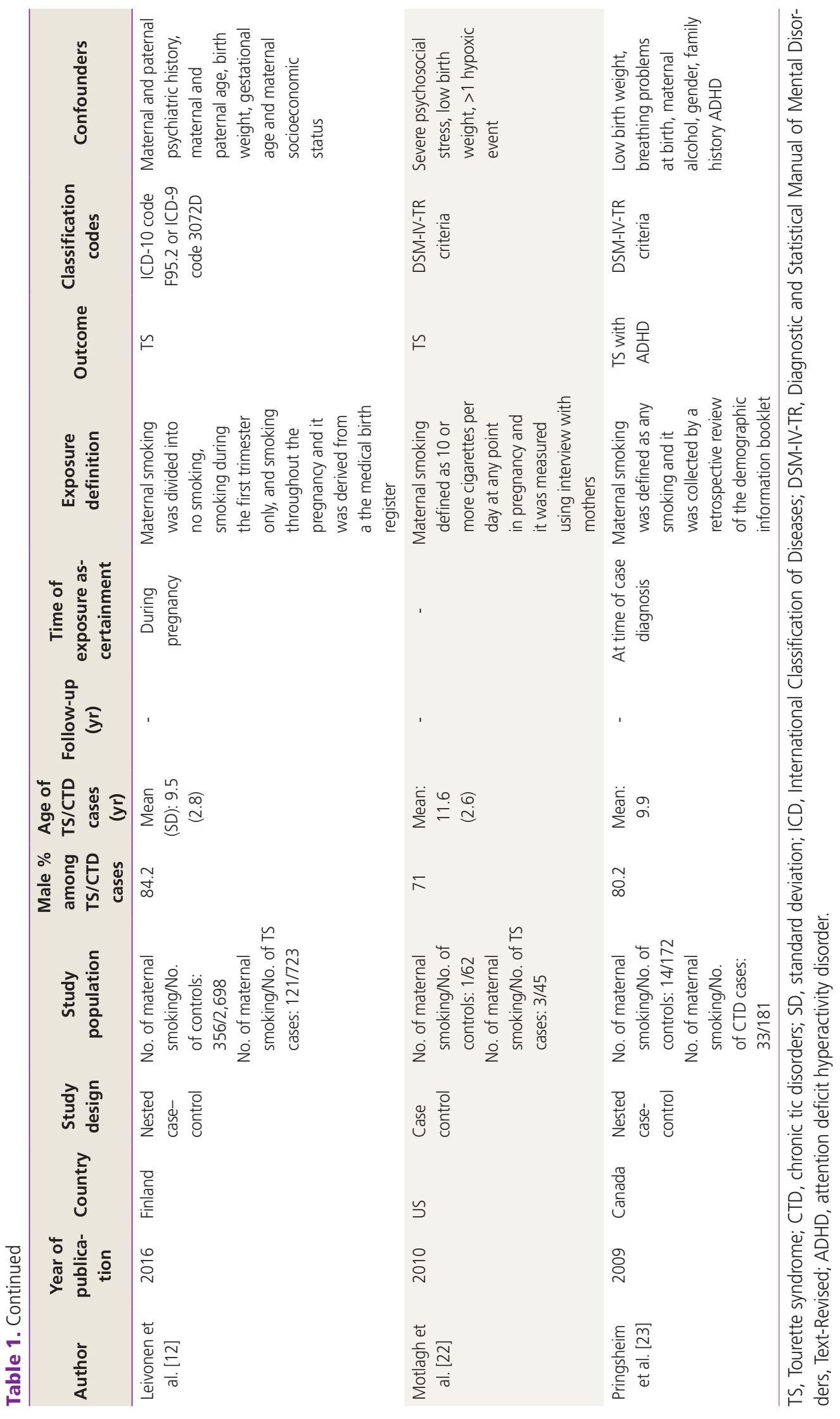




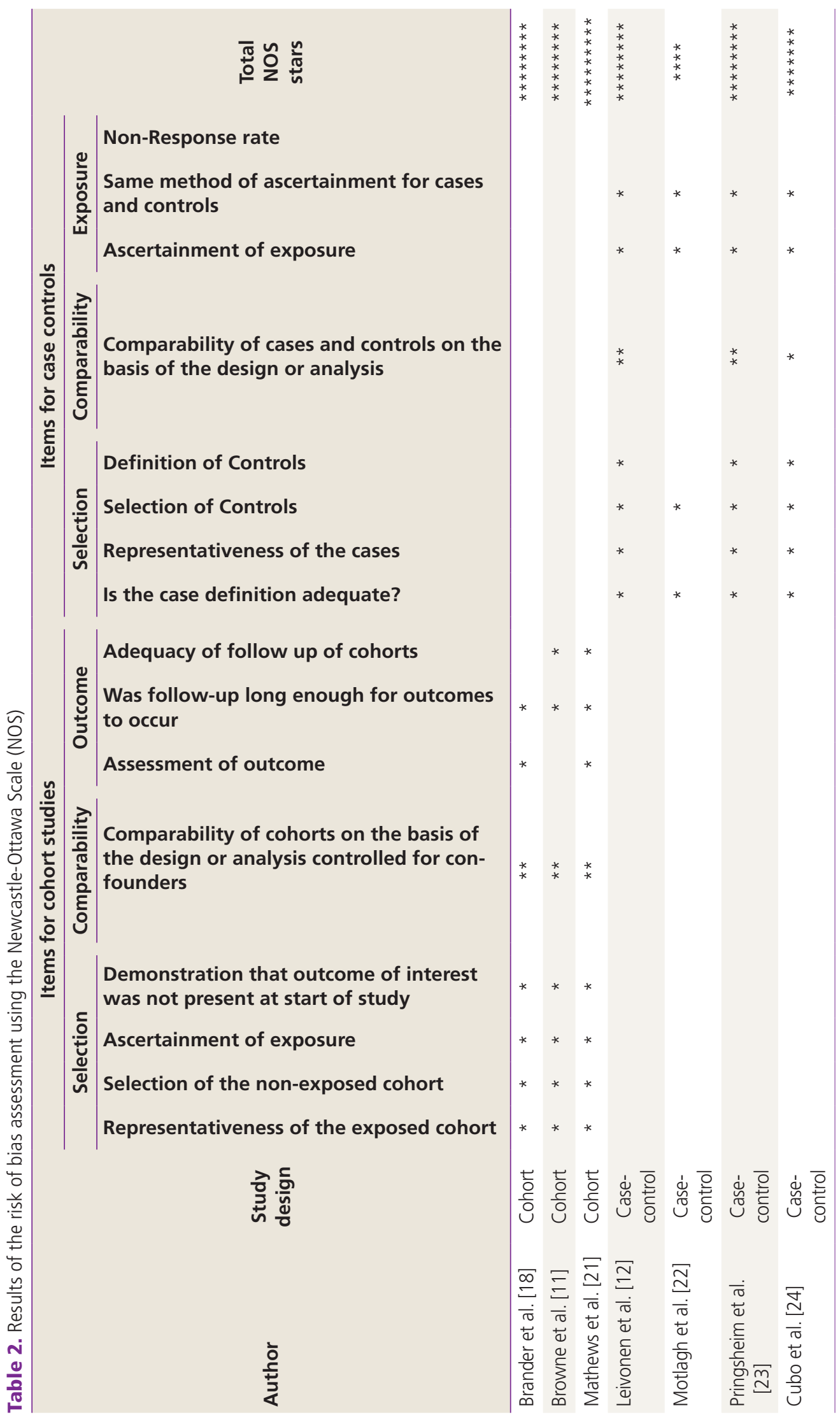




\title{
Obstetrics \& Gynecology Science
}

\author{
Erfan Ayubi, et al. MSDP and TS, CTD and TS/CTD
}

a low degree of bias (NOS stars $\geq 6$ ). Based on the NOS, the maximum number of stars achieved among the cohort studies was 9, given to the one by Mathews et al. [21]. Among case control studies, those by Leivonen et al. [12] and Pringsheim et al. [23] received the highest number of stars, 8 each (Table 2).

\section{Results of meta-analysis}

In individual studies, adjusted HRs or ORs were reported for the association between MSDP and TS or CTD in several scenarios. For example, in 2 studies by Brander et al. [18] and Browne et al. [11], the association between MSDP and TS/CTD has been examined alone or with other comorbid psychiatric conditions. In the study by Brander et al. [18], 2 adjusted HRs were reported for the association between TS and CTD and 2 categories of MSDP, 1-9 cigarettes per day and $\geq 10$ cigarettes per day; however, we pooled these 2 HRs. Finally, 7 adjusted HRs and ORs from 7 observational studies were included in the meta-analysis. The overall adjusted RR $(95 \% \mathrm{Cl})$ of MSDP on TS and CTD based on the fixedeffects model was $1.35(95 \% \mathrm{Cl}, 1.17-1.56) ; I^{2}=45.8 \%$; $P$ value $=0.08$ (Fig. 2). Corresponding figures based on the random-effects model were 1.44 (1.14-1.82) (forest plot not shown).
In terms of study design, the pooled RR $(95 \% \mathrm{Cl})$ from cohort studies was $1.30(1.11-1.52) ; l^{2}=35 \% ; P$-value $=0.21$; and the corresponding figure from case control studies was $1.65(1.16-2.35) ; I^{2}=54.2 \% ; P$-value $=0.09$. Stratification by the type of effect showed pooled associations when the $H R$ and $O R$ used for assessing the association were 1.32 $(1.12-1.56) ; I^{2}=65.2 \% ; P$-value $=0.09$, and 1.43 [1.10-1.85]; $\mathrm{I}^{2}=49.8 \%, P$-value $=0.09$, respectively. Furthermore, the resulting effect measures were stratified according to the outcome of interest. When excluding the OR from Pringsheim et al.'s study [23] (whose outcome was TS with attentiondeficit/hyperactivity disorder [ADHD]), the pooled RR was 1.32 $(1.14-1.52) ; I^{2}=38.4 \% ; P$-value $=0.15$. After removing the ORs from the studies by Leivonen et al. [12] and Motlagh et al. [22] (were outcome was only TS) as well as the OR from the study by Pringsheim et al. [23], the pooled RR was 1.33 $(1.14-1.55) ; I^{2}=53.3 \% ; P$-value $=0.09$.

\section{Publication bias}

The funnel plot showed that the included studies were distributed relatively symmetrically on both sides of the vertical line, which reflected the absence of potential publication bias. Moreover, the P-value for the 2 tests (Begg's and Egger's tests) was $>0.05$, indicating no publication bias (Fig. 3 ).

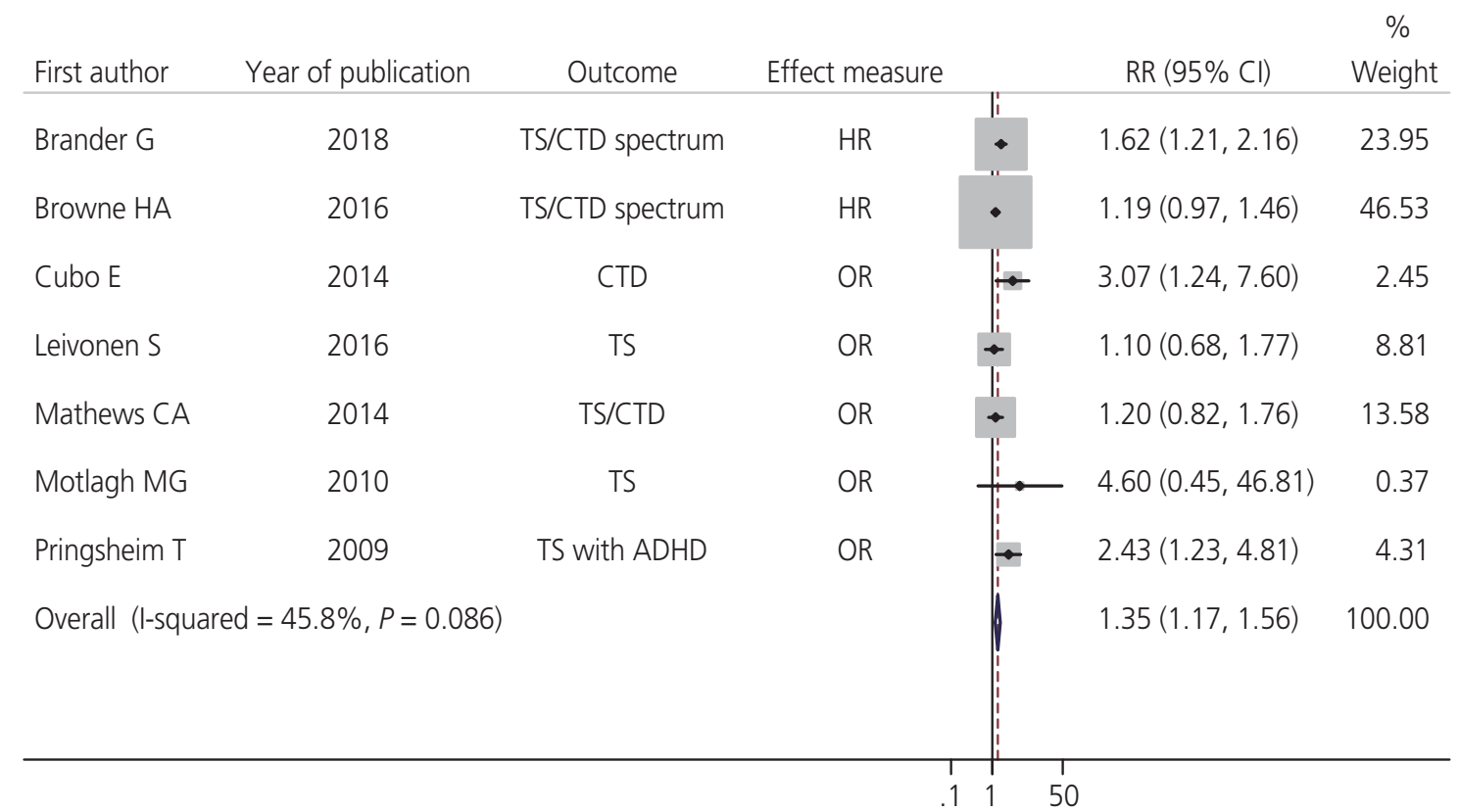

Fig. 2. Forest plot for the effect of maternal smoking during pregnancy (MSDP) on the risk of Tourette syndrome (TS) and chronic tic disorders (CTD) in offspring. ADHD, attention deficit hyperactivity disorder; $\mathrm{HR}$, hazard ratio; OR, odds ratio; $\mathrm{RR}$, relative risk; $\mathrm{Cl}$, confidence interval. 


\section{Obstetrics \& Gynecology Science}

Vol. 64, No. 1, 2021

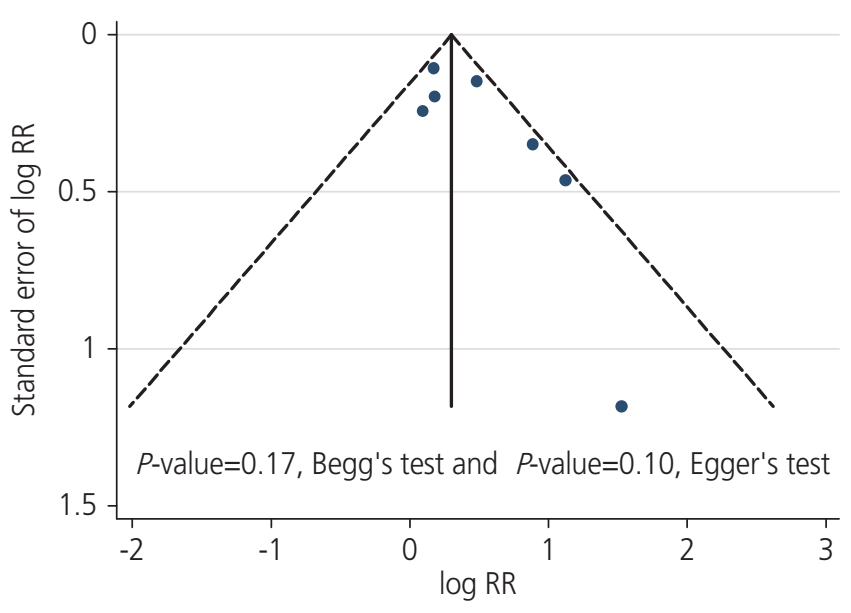

Fig. 3. Funnel plot and statistical tests for assessing the risk of publication bias of studies included in the meta-analyses. RR, relative risk.

\section{Discussion}

In this systematic review that led to the meta-analysis, a total of 641 potential articles were included. Finally, 7 adjusted RRs from 7 observational studies were pooled in the metaanalysis. The results showed that MSDP may be associated with a 35\% increase in the risk of TS and CTD among offspring.

Although the biological mechanisms underlying the link between MSDP and TS/CTD are still not well understood, the findings from animal studies suggest that in utero nicotine exposure may influence fetal brain development and have short- and long-term effects on function [25-31]. Specifically, nicotine exposure during early fetal development adversely affects the function of neurotransmitters in the brain and synaptic development, causing neuronal loss and cell death [25]. In addition, MSDP can lead to an increased risk of adverse outcomes such as preterm birth and low birth weight, which are known to be potential risk factors for TD/CTD [8,21].

An important consideration in this meta-analysis is that the majority of the included RRs are estimated from cohort studies (weight=84\%). Cohort studies are the "gold standard" among the observational epidemiological study designs, and meta-analyses of such studies can be considered as being at the top of the hierarchy of studies generating scientific evidence. In cohort studies, temporality between exposure and outcome can be well defined. In the cohort studies included
$[11,18,21]$ in this meta-analysis, information about MSDP was gathered mainly during pregnancy; however, in 2 of the case control studies included $[22,23]$ this information was collected at the time of case diagnosis, which may increase the risk of recall bias and subsequent biased estimates of effect for the association between MSDP and TS/CTD. Another factor to be considered is that the included cohort studies varied in confounder adjustment. For example, in the studies by Browne et al. [11] and Mathews et al. [21], the confounding factor of alcohol consumption during pregnancy was considered in the multivariable analysis; however, in the study by Brander et al. [18] this confounder was not included in the analysis. This differing approach to confounder adjustment among the cohort studies can influence the pooled estimation. Another issue with the included cohort studies is that they $[11,18,21]$ tried to adjust for many potential confounders in the MSDP-TS/CTD pathway.

We found the association between MSDP and TS/CTD to be similar to that in other meta-analyses that evaluated the association of MSDP with other neurodevelopmental disorders. For example, in 2 meta-analyses [32,33], an association between MSDP and ADHD has been identified, and in another meta-analysis [34], an association between MSDP and autism spectrum disorder in offspring has been documented.

It is important to consider the association between MSDP and TS/CTD in the presence of potential confounders. The risk of TS/CTD may be different for the offspring of mothers who smoked only in part of pregnancy compared to those whose mothers smoked during the whole pregnancy. For example, among the included studies, the one by Mathews et al. [20] showed that the effects of smoking in the last 2 months of pregnancy for TS and TS/CTD were 0.99 and 1.25 respectively, and these were different when compared to those reported by other included studies that assumed smoking in the whole pregnancy. A dose-response meta-analysis of cigarette dose and the risk of TS/CTD is needed when smoking is measured as a continuous variable. Previous literature offers a link between MSDP and TS and CTD disorders but is confounded by the presence of comorbidities [12,22].

Another source that could explain the heterogeneity among studies is the role of the interaction between MSDP and other risk factors. For example, in one review, maternal smoking and low birth weight were only 2 important determinants of TS [8] and in one study by Santangelo et al. [35] coffee, cigarettes, and alcohol exposure were combined as 


\section{Obstetrics \& Gynecology Science}

Erfan Ayubi, et al. MSDP and TS, CTD and TS/CTD

one single factor that was a risk factor TS. In another study by Bos-Veneman et al. [17], the association between MSDP and TS/ADHD is stronger in children with a family history of mental disorders than in those without a family history of mental disorders.

The present meta-analysis has several strengths and limitations. This study is the first meta-analysis that aimed to investigate the association between MSDP and TS/CTD among offspring, including mainly cohort studies and case control studies, with a relatively large sample sizes and low risk of bias. However, the number of studies included in the metaanalysis was 7 (3 cohort and 4 case-control studies); therefore, the effect of publication bias and small study effects on the results should be considered when interpreting the results.

Another limitation of this systematic review was the variety in the reported outcomes in the included studies. However, in addition to estimating a pooled measure of association, we reported a specific study measure of association for each outcome, for example TS/CTD spectrum, only TS, only CTD, TS/CTD, and TS with ADHD.

Moreover, this study may be accompanied by a degree of selection bias because of potentially missed studies indexed in databases such as Embase and the Cochrane Library, limiting full-text review to English language articles, or no systematic attempt to obtain gray literature.

\section{Conclusion}

The results of this systematic review and meta-analysis indicated that the MSDP may be associated with the risk of TS and CTD in offspring; therefore, the planning and implementation of educational and prevention programs for mothers to stop smoking, especially during pregnancy, seems to be necessary.

\section{Conflict of interest}

No potential conflict of interest relevant to this article was reported.

\section{Ethical approval}

None.

\section{Patient consent}

There is no need for patient consent in this review article.

\section{Funding information}

None.

\section{References}

1. Leckman JF, King RA, Bloch MH. Clinical features of Tourette syndrome and tic disorders. J Obsessive Compuls Relat Disord 2014;3:372-9.

2. Scahill L, Sukhodolsky DG, Williams SK, Leckman JF. Public health significance of tic disorders in children and adolescents. Adv Neurol 2005;96:240-8.

3. Yang J, Hirsch L, Martino D, Jette N, Roberts J, Pringsheim $\mathrm{T}$. The prevalence of diagnosed Tourette syndrome in Canada: a national population-based study. Mov Disord 2016;31:1658-63.

4. Pauls DL, Fernandez TV, Mathews CA, State MW, Scharf JM. The inheritance of Tourette disorder: a review. J Obsessive Compuls Relat Disord 2014;3:380-5.

5. Davis LK, Yu D, Keenan CL, Gamazon ER, Konkashbaev Al, Derks EM, et al. Partitioning the heritability of Tourette syndrome and obsessive compulsive disorder reveals differences in genetic architecture. PLoS Genet 2013;9:e1003864.

6. Georgitsi M, Willsey AJ, Mathews CA, State M, Scharf JM, Paschou P. The genetic etiology of Tourette syndrome: large-scale collaborative efforts on the precipice of discovery. Front Neurosci 2016;10:351.

7. Hoekstra PJ, Dietrich A, Edwards MJ, Elamin I, Martino D. Environmental factors in Tourette syndrome. Neurosci Biobehav Rev 2013;37:1040-9.

8. Chao TK, Hu J, Pringsheim T. Prenatal risk factors for Tourette syndrome: a systematic review. BMC Pregnancy Childbirth 2014;14:53. 


\section{Obstetrics \& Gynecology Science}

Vol. 64, No. 1, 2021

9. Krewski D, Barakat-Haddad C, Donnan J, Martino R, Pringsheim T, Tremlett $\mathrm{H}$, et al. Determinants of neurological disease: synthesis of systematic reviews. Neurotoxicology 2017;61:266-89.

10. Orlovska S, Vestergaard CH, Bech BH, Nordentoft M, Vestergaard M, Benros ME. Association of streptococcal throat infection with mental disorders: testing key aspects of the PANDAS hypothesis in a nationwide study. JAMA Psychiatry 2017;74:740-6.

11. Browne HA, Modabbernia A, Buxbaum JD, Hansen $\mathrm{SN}$, Schendel DE, Parner ET, et al. Prenatal maternal smoking and increased risk for Tourette syndrome and chronic tic disorders. J Am Acad Child Adolesc Psychiatry 2016;55:784-91.

12. Leivonen S, Chudal R, Joelsson P, Ekblad M, Suominen A, Brown AS, et al. Prenatal maternal smoking and Tourette syndrome: a nationwide register study. Child Psychiatry Hum Dev 2016;47:75-82.

13. Moher D, Liberati A, Tetzlaff J, Altman DG; PRISMA Group. Preferred reporting items for systematic reviews and meta-analyses: the PRISMA statement. PLoS Med 2009;6:e1000097.

14. Stang A. Critical evaluation of the Newcastle-Ottawa scale for the assessment of the quality of nonrandomized studies in meta-analyses. Eur J Epidemiol 2010;25:603-5.

15. Cummings $P$. The relative merits of risk ratios and odds ratios. Arch Pediatr Adolesc Med 2009;163:438-45.

16. Higgins JP, Thompson SG, Deeks JJ, Altman DG. Measuring inconsistency in meta-analyses. BMJ 2003;327:55760.

17. Bos-Veneman NG, Kuin A, Minderaa RB, Hoekstra PJ. Role of perinatal adversities on tic severity and symptoms of attention deficit/hyperactivity disorder in children and adolescents with a tic disorder. J Dev Behav Pediatr 2010;31:100-6.

18. Brander $G$, Rydell M, Kuja-Halkola R, Fernández de la Cruz L, Lichtenstein $P$, Serlachius $E$, et al. Perinatal risk factors in Tourette's and chronic tic disorders: a total population sibling comparison study. Mol Psychiatry 2018;23:1189-97.

19. Cubo E, Hortigüela M, Roldan S, Ciciliani C, Lopez P, Velasco $L$, et al. Prenatal and perinatal morbidity in children with tic disorders. A mainstream school-based population study in central Spain (P02.066). Neurology
2013;80:P02.066.

20. Mathews CA, Bimson B, Lowe TL, Herrera LD, Budman $C L$, Erenberg $G$, et al. Association between maternal smoking and increased symptom severity in Tourette's syndrome. Am J Psychiatry 2006;163:1066-73.

21. Mathews CA, Scharf JM, Miller LL, Macdonald-Wallis C, Lawlor DA, Ben-Shlomo Y. Association between pre- and perinatal exposures and Tourette syndrome or chronic tic disorder in the ALSPAC cohort. Br J Psychiatry 2014;204:40-5.

22. Motlagh MG, Katsovich L, Thompson N, Lin H, Kim YS, Scahill $L$, et al. Severe psychosocial stress and heavy cigarette smoking during pregnancy: an examination of the pre- and perinatal risk factors associated with ADHD and Tourette syndrome. Eur Child Adolesc Psychiatry 2010;19:755-64.

23. Pringsheim T, Sandor P, Lang A, Shah P, O'Connor P. Prenatal and perinatal morbidity in children with Tourette syndrome and attention-deficit hyperactivity disorder. J Dev Behav Pediatr 2009;30:115-21.

24. Cubo E, Hortigüela M, Jorge-Roldan S, Ciciliani SE, Lopez P, Velasco L, et al. Prenatal and perinatal morbidity in children with tic disorders: a mainstream school-based population study in central Spain. Tremor Other Hyperkinet Mov (N Y) 2014;4:272.

25. Blood-Siegfried J, Rende EK. The long-term effects of prenatal nicotine exposure on neurologic development. J Midwifery Womens Health 2010;55:143-52.

26. Bublitz MH, Stroud LR. Maternal smoking during pregnancy and offspring brain structure and function: review and agenda for future research. Nicotine Tob Res 2012;14:388-97.

27. Dalsgaard S, Waltoft BL, Leckman JF, Mortensen PB. Maternal history of autoimmune disease and later development of Tourette syndrome in offspring. J Am Acad Child Adolesc Psychiatry 2015;54:495-501.e1.

28. Ernst M, Moolchan ET, Robinson ML. Behavioral and neural consequences of prenatal exposure to nicotine. J Am Acad Child Adolesc Psychiatry 2001;40:630-41.

29. Liu J, Lester BM, Neyzi N, Sheinkopf SJ, Gracia L, Kekatpure $\mathrm{M}$, et al. Regional brain morphometry and impulsivity in adolescents following prenatal exposure to cocaine and tobacco. JAMA Pediatr 2013;167:348-54.

30. Martino D, Zis $P$, Buttiglione $M$. The role of immune mechanisms in Tourette syndrome. Brain Res 


\section{Obstetrics \& Gynecology Science}

Erfan Ayubi, et al. MSDP and TS, CTD and TS/CTD

2015;1617:126-43.

31. Steeves TD, Ko JH, Kideckel DM, Rusjan P, Houle S, Sandor $P$, et al. Extrastriatal dopaminergic dysfunction in Tourette syndrome. Ann Neurol 2010;67:170-81.

32. He Y, Chen J, Zhu LH, Hua LL, Ke FF. Maternal smoking during pregnancy and ADHD: results from a systematic review and meta-analysis of prospective cohort studies. J Atten Disord 2020;24:1637-47.

33. Huang L, Wang Y, Zhang L, Zheng Z, Zhu T, Qu Y, et al. Maternal smoking and attention-deficit/hyperactivity disorder in offspring: a meta-analysis. Pediatrics 2018;141:e20172465.

34. Jung $Y$, Lee AM, McKee SA, Picciotto MR. Maternal smoking and autism spectrum disorder: meta-analysis with population smoking metrics as moderators. Sci Rep 2017;7:4315.

35. Santangelo SL, Pauls DL, Goldstein JM, Faraone SV, Tsuang MT, Leckman JF. Tourette's syndrome: What are the influences of gender and comorbid obsessive-compulsive disorder? J Am Acad Child Adolesc Psychiatry 1994;33:795-804. 


\section{Obstetrics \& Gynecology Science}

Vol. 64, No. 1, 2021

Appendix 1. Search strategy

\section{PubMed $(n=10)$}

("tourette syndrome" [MeSH Terms] OR "tic disorders" [MeSH Terms] OR tics [MeSH Terms] OR " Tourette " [All Fields] OR " Tourette Syndrome " [All Fields] OR " Tourette's Syndrome " [All Fields] OR "Tourette's disorder" [All Fields] OR "Tourette's Disease" [All Fields] OR " Gilles de la Tourette syndrome" [All Fields] OR "tic disorder" [All Fields] OR "tic disorders" [All Fields] OR " chronic tic" [All Fields] OR "tic" [All Fields] OR "tics" [All Fields])

AND

(smoking [MeSH Terms] OR "parental smoking" [All Fields] OR "parent smoking" [All Fields] OR "parental smoke" [All Fields] OR "maternal smoking" [All Fields] OR "maternal smoke" [All Fields] OR "paternal smoking" [All Fields] OR "paternal smoke" [All Fields] OR "mother smoking" [All Fields] OR "mother smoke" [All Fields])

AND

(Pregnancy [Mesh Terms] OR Pregnant [All Fields] OR Gestational [All Fields])

AND

(Child [Mesh Terms] OR Pediatrics [Mesh Terms] OR Adolescent [Mesh Terms] OR Children [All Fields] OR offspring [All Fields])

\section{Web of Science $(n=573)$}

TITLE: ("tic disord*") OR TITLE: ("tourette *") AND TITLE: ("maternal smoking") OR TITLE: ("parental smoking") AND TITLE: ("child*") AND TITLE: ("pregnan*")

Timespan: All years. Indexes: SCI-EXPANDED, SSCI, CPCI-S, CPCI-SSH, ESCI.

\section{Scopus $(n=58)$}

( TITLE-ABS-KEY ( "tourette syndrome") OR TITLE-ABS-KEY ( "tourette *") OR TITLE-ABS-KEY ( "tic disorders") OR TITLEABS-KEY ("tic*") AND TITLE-ABS-KEY ( "maternal smoking") OR TITLE-ABS-KEY ( "parental smoking" ) OR TITLE-ABSKEY (smoking) AND TITLE-ABS-KEY ( pregnan*)) 